\title{
Robots Used Today that we Did Not Expect 20 Years Ago (from the Editorial Board Members)
}

\author{
Tauseef Gulrez ${ }^{1}$
}

Received: 12 June 2021 / Accepted: 12 June 2021 / Published online: 1 July 2021

(C) Crown 2021

With every passing day, robots either autonomous or having humans-in-the-loop, are becoming ubiquitous property of this world. From deep-ocean exploration to space-navigation and exploration, replacing a surgeon in the operating room or acting as a chauffeur of a car, robotics influence is growing daily. Journal of Intelligent and Robotic Systems is a much-required timely forum informing the latest technical advancements for the critical issues in robotics.

Robotics has grown enormously in the last two decades. The world of open source collaborative robotic platforms, together with shared artificial intelligence (AI) tools to solve navigation, control, perception and manipulation tasks has pushed robotics expansion further along.

In the universe of biological robotics, DNA-robotics will soon deliver medicinal aid inside our bodies, while detecting the deadly pathogens and timely informing our immune system [1]. These robots may secure humanity from future COVID-19 like pandemics and deadly viruses. On the fronts of brain-robotinterface, a new brain stimulation mechanism can evoke tactile sensations for the people with disabilities using a braincontrolled prosthetic arm [2]. Rehabilitation robotics is undoubtedly a growing field, extending (bidirectional) humans' physical abilities, robotics may answer one day the brain adaptations to this extra-personal robotic body part.

The molecular and micro-robotics have developed robotic particles less than 1/30th of a hair diameter. Capable of navigation, these miniaturised robots have still to learn perceptually and sense their surrounding environment.

Moreover, recent progress in quantum computing has confirmed its promise to deliver a huge potential for adaptive learning for real-life applications. Hence, the idea of merging it with the fields of robotics: on one hand, artificial intelligence with its

Tauseef Gulrez

gtauseef@ieee.org

1 Aerospace Division, Defence Science and Technology Group, Melbourne, Fishermans Bend, Victoria 3207, Australia autonomous machines; on the other hand, quantum physics with its powerful algorithms. Over the past few years, many scientists have started to investigate how to bridge robotics and quantum computing, to study how quantum mechanics can be used for robotic learning. Encouraging results [3] have been shown but robots are still incapable of learning faster an essential element of increasingly complex autonomous robotics.

Recent advancements in artificial intelligence (AI) and computer vision are acting as a catalyst for autonomous robotics research. To make autonomous self-driving cars safer, AI based computer vision capture surrounding environment and records the vehicle status e.g. road conditions, steering wheel angle, visibility, weather and speed [4]. The AI based on a recurrent neural network (RNN), learns to recognize driving and surrounding patterns with the data. If the AI detects a pattern in a new driving situation that the control system was unable to handle in the past, the driver will be warned in advance of a possible critical situation, within seconds.

Interestingly, humanoid robotic paradigms are being used to test the psychological basis of the human mind. Researchers have argued that the established feedforward models of sensorimotor control are not consistent with the human psychological experimental studies conducted by several research groups. Robotic paradigms have shown that brain mechanisms do not require motor planning but utilise the perceptual consequences of action for motor control. This means that pure computational models are limited in their roles to the physics of the body and environment. Therefore, robotic devices are necessary in-order to advance testing of the human psychological studies [5].

For robotics to make a clear and positive impact to society, I personally believe skies are not the limits rather robotics has no boundaries, collective wisdom can only come with collective action. The time is ripe to pull together an effective robotic community.

Sincerely,

Dr. Tauseef Gulrez 


\section{References}

1. Huang, C. M., Kucinic, A., Johnson, J. A., Su, H. J., \& Castro, C. E. (2021). Integrated computer-aided engineering and design for DNA assemblies. Nature Materials, 1-8

2. Flesher, N.S., Downey, E.J., Weiss, M.J., Hughes, L.C., Herrera, J.A., Tyler-Kabara, C.E., Boninger, L.M., Collinger, L.J., Gaunt, A.R.: A brain-computer interface that evokes tactile sensations improves robotic arm control. Science. 2021, 831-836 (2021). https:// doi.org/10.1126/science.abd0380

3. Saggio, V., Asenbeck, B.E., Hamann, A., Strömberg, T., Schiansky, P., Dunjko, V., Friis, N., Harris, N.C., Hochberg, M., Englund, D., Wölk, S., Briegel, H.J., Walther, P.: Experimental quantum speed-up in reinforcement learning agents. Nature. 591(7849), 229-233 (2021)

4. Kuhn, B.C., Hofbauer, M., Petrovic and Steinbach, E.: Introspective failure prediction for autonomous driving using late fusion of state and camera information. IEEE Trans. Intell. Transp. Syst. 1-15 (2020). https://doi.org/10.1109/TITS.2020.3044813

5. Sandhu, S., Gulrez, T., Mansell, W.: Behavioral anatomy of a hunt: using dynamic real-world paradigm and computer vision to compare human user-generated strategies with prey movement varying in predictability. Atten. Percep. Psychophys. 82, 3112-3123 (2020)

Publisher's Note Springer Nature remains neutral with regard to jurisdictional claims in published maps and institutional affiliations.
Tauseef Gulrez received his masters by research in computer engineering from University of Technology, Sydney in 2005, a neuroengineering predoctoral fellowship from Northwestern University, Chicago, IL, USA in 2006 and a Ph.D. in robotics and artificial intelligence from Department of Computing, Macquarie University, Sydney, Australia, in September 2008. He was a postdoctoral research fellow at Mechatronics and Haptic Interface Lab, Rice University, Houston, TX, USA, Virtual Interactive Simulations of Reality Lab, Macquarie University, Sydney and Learning Affect Technologies Lab, University of Sydney, Australia. He held positions of senior research fellow at the Center for Robotics and Autonomous Systems, University of Salford, Manchester, Salford Royal NHS Foundation Trust, Manchester, Biorobotics Lab of University of Washington, Seattle and Cognitive Engineering Lab of Australian Defence Force Academy (ADFA), Canberra. Presently, he is working as a robotics and artificial intelligence research scientist at Aerospace Division, Defence Science and Technology, Group, Melbourne. He is an author of a book, has published more than 50 research journal and conference articles. He is an inventor of cutaneous haptic interface used for a surgical robot, for which he received his US patent in 2017. He is a recipient of 3 international research grants. His main research interests include robotics, $\mathrm{AI}$, computer vision and behavioural neuroscience. 